\title{
Endothelial Microparticles and Vascular Endothelial Growth Factor in Patients With Head and Neck Cancer Undergoing Radiotherapy or Radiochemotherapy
}

\author{
EWA SIERKO $^{1,2}$, MONIKA SOBIERSKA ${ }^{1,3}$, EWA ZABROCKA $^{4}$, JOANNA KRUSZEWSKA $^{1}$, \\ MARTA MYŚLIWIEC ${ }^{1}$, ALINA LIPSKA ${ }^{3}$, PIOTR RADZIWON ${ }^{3}$ and MAREK Z. WOJTUKIEWICZ ${ }^{1}$ \\ ${ }^{1}$ Department of Oncology, Medical University of Bialystok, Bialystok, Poland; \\ ${ }^{2}$ Department of Radiotherapy, Comprehensive Cancer Center in Bialystok, Bialystok, Poland; \\ ${ }^{3}$ Regional Centre for Transfusion Medicine, Bialystok, Poland; \\ ${ }^{4}$ Department of Medicine, Stony Brook University, Stony Brook, NY, U.S.A.
}

\begin{abstract}
Background: Endothelial microparticles (EMPs) released from activated or apoptotic endothelial cells may play a role in coagulation and thrombus formation. However, there is insufficient evidence regarding the impact of EMPs on angiogenesis in patients with cancer. Materials and Methods: Sixteen patients with head and neck cancer $(H N C) \quad$ undergoing radiotherapy/radiochemotherapy $(R T / R C T)$ and 10 healthy controls were studied. Serum EMPs were counted by flow cytometry, and vascular endothelial growth factor (VEGF) was measured by enzymelinked immunosorbent assay (ELISA). Results: The mean EMP level was significantly higher in patients with HNC before $R T / R C T(1,601 \pm 1,479 E M P / \mu l)$ compared to the control group $(782 \pm 698 \mathrm{EMP} / \mu \mathrm{l})$. The number of EMPs was not notably increased after RT/RCT (1,629 $769 \mathrm{EMP} / \mu \mathrm{l})$. There was no significant correlation between the plasma EMP number and concentration of VEGF before $(r=0.131$; $p=0.625), 1$ day after $(r=-0.042, p=0.874)$, nor 3 months after RT/RCT ( $r=0.454, p=0.076)$. Conclusion: Released EMPs may not influence promotion of neovascularization in patients with $H N C$.
\end{abstract}

Endothelial microparticles (EMPs) are vesicular structures with a diameter from 1 to $2 \mu \mathrm{m}$ which are shed from activated or apoptotic endothelial cells (ECs) (1). The density of EMPs in

This article is freely accessible online.

Correspondence to: Ewa Sierko, Department of Oncology, Medical University in Bialystok, Ogrodowa 12, 15-027 Bialystok, Poland. Tel: +48 856646734, Fax: +48 856646783, e-mail: ewa.sierko@iq.pl

Key Words: Endothelial microparticles, flow cytometry, vascular endothelial growth factor, head and neck cancer, angiogenesis, radiation therapy. the blood of healthy individuals ranges from $1-70 \times 10^{3} / \mathrm{ml}(1)$. Knowledge about EMP formation has been obtained from experiments conducted on isolated or cultured ECs, whereas in vivo mechanisms involved in EMP generation still remain unclear. Inflammatory cytokines $[e . g$. tumor necrosis factor- $\alpha$, (TNF- $\alpha$ )] bacterial lipopolysaccharides, reactive oxygen species, thrombin, camptothecin and chemotherapy were reported to induce EMP generation $(1,2)$.

EMPs express a large variety of molecules representative of their parent cells (3). Their composition differs depending on the cells they originate from and the type of stimulus leading to their formation (4). On their surface, EMPs bear phospholipids, membrane receptors such as endothelial protein $\mathrm{C}$ receptor, thrombomodulin, tissue factor, adhesion molecules such as intercellular cell adhesion molecule-1, platelet-EC adhesion molecule, endothelial selectin and P-selectin (3). In addition, EMPs harbor enzymes such as matrix metalloproteinase, nicotinamide adenine dinucleotide phosphate oxidase, urokinase plasminogen activator and its receptor, and growth factor receptors (3).

The density of EMPs is elevated in various clinical settings. Activated or apoptotic EC-derived EMPs are a marker of endothelial damage and their level was found to be increased in the blood of obese women, patients with terminal stage renal failure or multiple sclerosis (5-7). Moreover, it has been documented that EMPs contribute to initiation of blood coagulation and support thrombus formation $(3,8,9)$. Furthermore, a higher level of EMPs was found in patients with hematological disorders e.g. lupus anticoagulant, sickle cell disease, anti-phospholipid syndrome and venous thromboembolism, than in healthy individuals $(3,9,10)$. In addition, an increased level of EMPs was noted in patients with cancer (11).

The current standard of treatment for patients with newlydiagnosed head and neck cancer (HNC) is surgery followed by radiotherapy (RT) or radiochemotherapy (RTC), RT alone 
or RTC. Ionizing radiation affects both cancer and normal cells within the irradiated volume causing acute postradiation reaction and patients experience symptoms associated with tissue damage for a few weeks, months or even years after RT. The symptoms caused by RT begin 2-3 weeks into the treatment, with the greatest intensity at the end of RT and soon after its completion. They usually resolve after 6-8 weeks after treatment, and are absent after 3 months in most patients (12). Injury caused by RT to normal tissue might be a significant factor leading to EMP generation from endothelium. Moreover, radiation stimulates angiogenesis by induction of nitric oxide synthesis in ECs, which leads to temporary hypoxia within a tumor, and induces further synthesis of vascular endothelial growth factor (VEGF), which protects ECs from the cytotoxic effect of ionizing radiation (13-15).

It has been reported that the number of EMPs might increase in diseases associated with endothelial damage, such as hematological disorders, or among patients undergoing specific treatment (e.g. with statins) $(16,17)$. Nevertheless, there are only scant data concerning the influence of $\mathrm{RT} / \mathrm{RCT}$ on EMP formation in the process of EC apoptosis or activation. A few studies documented that EMPs may exhibit pro-angiogenic properties $(11,18)$, but little is known about the impact of EMPs on tumor angiogenesis in patients with HNC during RT/RCT. The aim of this study was to investigate the potential contribution of EMPs to angiogenesis in patients with HNC undergoing RT/RCT.

\section{Materials and Methods}

This study was performed on a group of 16 patients with HNC, (five females) aged 31-70 years, with histopathologically-diagnosed with clinical stage IIB-IVA squamous cell carcinoma. Patients with conditions known or suspected to increase the EMP level, such as lupus anticoagulant, anti-phospholipid syndrome, venous thromboembolism, metabolic syndrome, diabetes mellitus, stroke as well as advanced stages of renal failure, were excluded from the study. Patient characteristics are shown in Table I. The control group consisted of 10 healthy individuals (seven females). The study protocol was approved by the Bioethics Committee of Medical University in Bialystok, Poland according to the Guidelines for Good Clinical Practice (approval number - R-I-002/376/2010). Written informed consent was obtained from the patients.

Blood samples were collected before RT (no acute radiation reaction observed), 1 day after its cessation (clinically overt radiationinduced inflammation) and 3 months after the treatment (when acute radiation reaction had resolved). Patients were treated with RT alone, adjuvant RT, or RT combined with chemotherapy $\left(100 \mathrm{mg} / \mathrm{m}^{2}\right.$ cisplatin every 21 days or $40 \mathrm{mg} / \mathrm{m}^{2}$ every week). Intensity-modulated RT with high-energy 6-MV photon beams to a total dose of 60-67.5 Gy in 30 fractions (2-2.25 Gy per day) was administered to the tumor volume/tumor bed and lymphatic regions of the neck.

Venous blood was collected in Monovette-Sarstedt tubes containing 3.8\% sodium citrate (Becton Dickinson, Franklin Lakes, NJ, USA) using 21-gauge needles (BD) in aliquots of $5 \mathrm{ml}$ from all
Table I. Baseline characteristics of patients with head and neck cancer $(n=16)$

\begin{tabular}{lc}
\hline Clinical factor & $\mathrm{n}(\%)$ \\
\hline Gender & \\
Female & $5(31)$ \\
Male & $11(69)$ \\
Site of cancer & \\
Lip, tongue & $2(12,5)$ \\
Floor of oral cavity and palate & $4(25)$ \\
Hyopharynx & $3(19)$ \\
Tonsil & $2(12,5)$ \\
Indeterminate part of oral cavity & $5(31)$ \\
Clinical stage* & \\
II B & $1(6)$ \\
III & $4(25)$ \\
IV A & $11(69)$ \\
Grade & \\
1 & $2(12.5)$ \\
2 & $12(75)$ \\
3 & $2(12.5)$ \\
Treatment & $7(44)$ \\
RT alone & $9(56)$ \\
RCT & \\
\hline
\end{tabular}

*According to TNM classification (20), RT: radiotherapy, RCT: radiochemotherapy.

Table II. Level of endothelial microparticles (EMPs) in patients with head and neck cancer undergoing radiotherapy $(R T)$ or radiochemotherapy $(R C T)$ and in a control group of healthy participants.

\begin{tabular}{lcc}
\hline Time of assessment & \multicolumn{2}{c}{ EMPs $[\mathrm{n} / \mu \mathrm{l}]$} \\
\cline { 2 - 3 } & Median & Mean \pm SD \\
\hline Control group & 1,141 & $782 \pm 698$ \\
Patients & 1,402 & $1,601 \pm 1,479^{*}$ \\
Before RT/RCT & 1,985 & $1,629 \pm 769^{*}$ \\
One day after RT/RCT & 1,496 & $1,238 \pm 374$ \\
Three months after RT/RCT & & \\
\hline
\end{tabular}

*Significantly different from the control group $(p<0.05)$.

participants. Isolation of EMPs from whole blood required centrifugation for $15 \mathrm{~min}$ at $500 \times g$ to remove erythrocytes and leukocytes and to prepare platelet-rich plasma. This was then further centrifuged for $5 \mathrm{~min}$ at $14,000 \times g$ to obtain platelet-poor plasma. Both steps of centrifugation were performed at room temperature. Plasma samples were not frozen because of the harmful influence of low temperature on EMP vitality and they were analyzed 1 hour after blood collection.

EMPs were defined as particles in size below $1.5 \mu \mathrm{m}$ bearing antigens CD31 and CD62E, and were quantified by flow cytometry using a panel of monoclonal antibodies to CD31, CD62E, and 


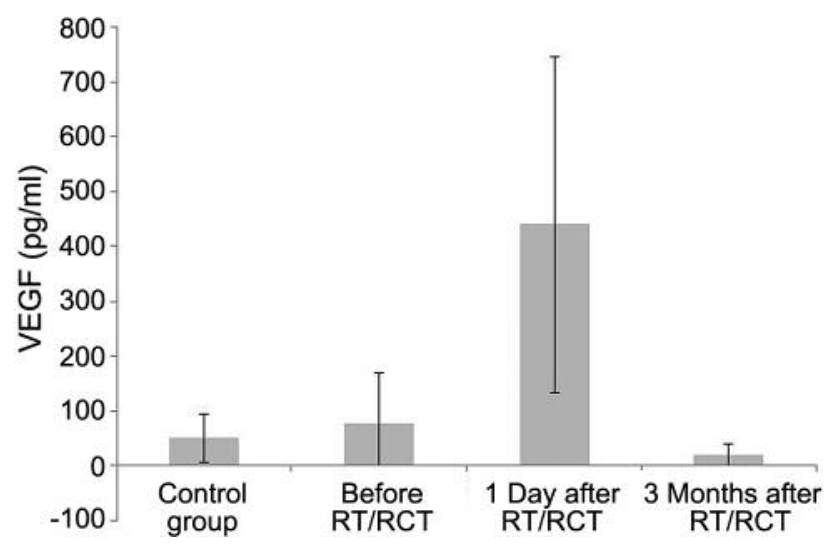

Figure 1. Levels of plasma vascular endothelial growth factor (VEGF) in patients with head and neck cancer undergoing radiotherapy $(R T)$ or radiochemotherapy $(R C T)$ and in the control group of healthy individuals.

CD42b; monoclonal antibody to CD42b was used to exclude EMPs released from platelets and megakaryocytes. The following monoclonal antibodies were used in EMP quantification: fluorescein isothiocyanate (FITC)-labeled CD31, allophycocyanin (APC)labeled CD62E, phycoerythrin (PE)-Cy5-labeled anti-human $\mathrm{CD} 42 \mathrm{~b}$, and kappa isotype control: FITC-labeled mouse IgG1, APClabeled mouse IgG, PE-Cy5-labeled mouse IgG1. Moreover, Cell WASH, FASC Lysing Solution, phosphate-buffered saline wash buffer, and FASC Flow Shealth Fluid were utilized in the study. All reagents were purchased from Becton Dickinson, Franklin Lakes, NJ, USA.

EMPs were analyzed by flow cytometry using FACS Calibur (Becton Dickinson) at the Regional Center for Transfusion Medicine in Bialystok, Poland, according to a protocol used in a previous study (19). The EMP gate was established using fluorospheres of known diameter (TruCOUNT; Becton Dickinson) and set to record fluorescent events at $<1.0 \mu \mathrm{m}$. Flow cytometry counts of EMPs were converted to the number of EMPs per $\mu$ using a standard formula.

The concentration of VEGF was measured using VEGF Quantikine ELISA, R\&D Systems, Minneapolis, MN, USA. Measurement of VEGF concentration in the blood of patients with HNC was performed using $5 \mathrm{ml}$ of venous blood collected into Monovette-Sarstedt tubes containing 3.8\% sodium citrate (Becton Dickinson). Blood samples were centrifuged for $15 \mathrm{~min}$ at $1,500 \times g$ to produce plasma, which was subsequently frozen $\left(\leq-20^{\circ} \mathrm{C}\right)$. The VEGF concentration was analyzed with ELISA Elx808 (BioTek) at the Department of Oncology, Medical University of Bialystok, Poland.

Statistical analysis. Measured values of EMPs are expressed as the mean, median and standard deviation as the counts were not normally distribution (Shapiro-Wilk test). Wilcoxon rank-sum test for dependent data was used to evaluate the significance of differences between EMP counts at different time-points of the study. The data obtained (EMP count and VEGF level) were tested for correlation using Spearman test. Statistical significance was defined as $p<0.05$. Analyses were performed using the statistical package STATISTICA 10 (Dell Software, Round Rock, TX, USA) and Microsoft Excel 2010 (Microsoft, Redmond, WA, USA).
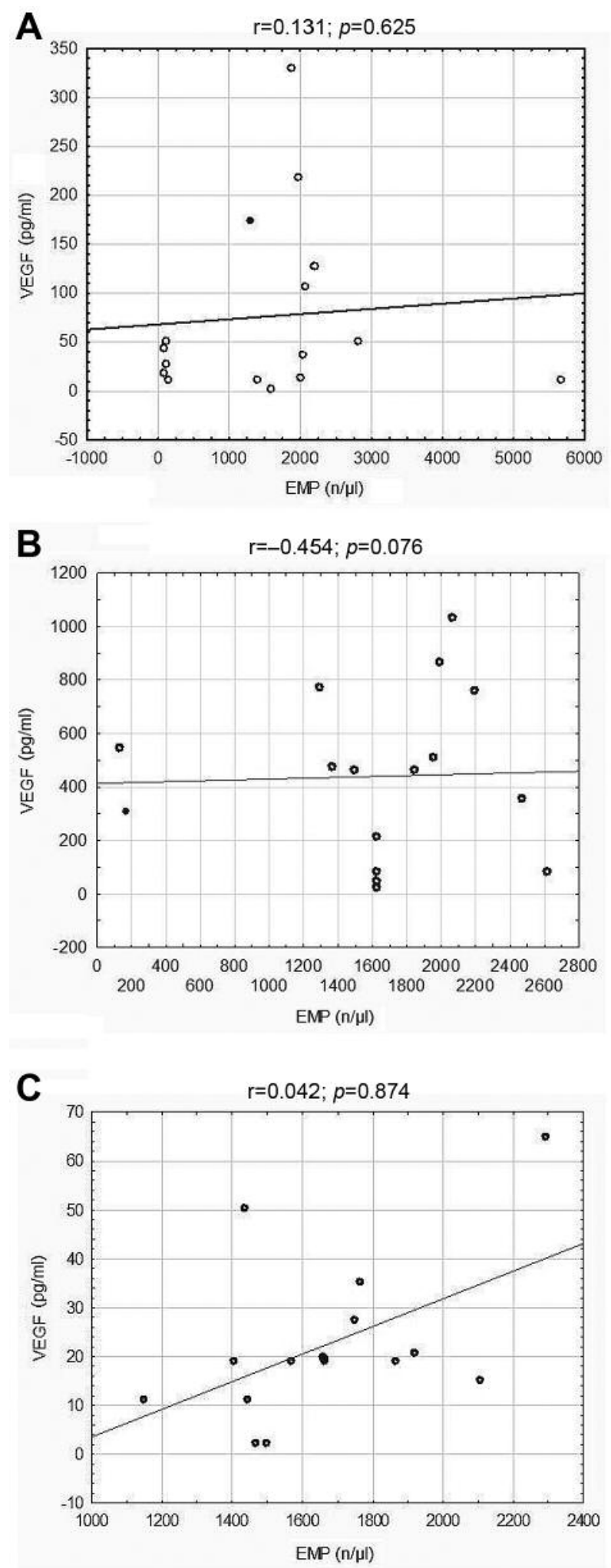

Figure 2. Correlation between number of endothelial microparticles (EMPs) and concentration of vascular endothelial growth factor (VEGF) in the plasma of patients with head and neck cancer before $(A)$ and at 1 day (B) and 3 months $(C)$ after radiotherapy or radiochemotherapy $(R T / R C T)$. 


\section{Results}

EMPs in patients with HNC. A significantly higher EMP count was observed in patients with HNC before RT/RCT and 1 day after the treatment by more than twofold in comparison to the control group (782 \pm 698 EMPs/ $\mu 1, p<0.05)$ (Table II). However, 3 months after the treatment, the number of EMPs was not significantly increased in comparison to that observed in healthy individuals. Furthermore, there were no significant differences between EMP counts before treatment, and at 1 day and 3 months after radiation cessation. Of note, no significant difference was demonstrated in EMPs counts in plasma of patients with HNC undergoing RT alone, RT combined with chemotherapy, or postoperative RT.

VEGF concentration in patients with HNC. No significant difference in VEGF concentration in plasma of patients with HNC before RT/RCT was observed compared to the control group ( $p>0.05$ ) (Figure 1). Interestingly, the VEGF level was 6-fold higher 1 day after RT/RCT compared to that in both the control group and that before RT/RCT $(p<0.05)$. Moreover, the VEGF concentration 3 months after treatment was significantly lower compared to that before RT and 1 day after RT completion $(p<0.05)$.

EMPs and VEGF. No correlation was found between EMP concentration and VEGF level in the plasma of patients with HNC before RT/RCT ( $\mathrm{r}=0,131 ; p=0,625)$ and 3 months after the treatment $(\mathrm{r}=-0,042, p=0,874)$. However, there was borderline significant negative correlation between EMP count and VEGF level 1 day after RT/RCT ( $r=-0.454$, $p=0.076)$ : increased VEGF concentration was accompanied by a decrease in EMP level (Figure 2).

\section{Discussion}

It was recently reported that ionizing radiation leads to different EC responses in vitro (21). After RT, ECs in cancer vasculature have less ability to form blood vessels as a result of radiation-induced injuries which destroy the integrity of vascular structure. However, ECs in non-cancerous tissue are stimulated to develop blood vessels after radiation.

The influence of EMPs on angiogenesis is not fully understood. It has been suggested that tissue factor-positive procoagulant microparticles released from ECs into the circulation may trigger venous thromboembolism in patients with cancer and contribute to a variety of pathological processes, such as metastasis, tumor growth and tumor angiogenesis. Angiogenesis is required for invasive tumor growth and metastasis and constitutes an important factor in cancer progression. In vitro studies demonstrated that the involvement of EMPs in angiogenesis is still a controversy.
Mezentsev et al. reported that EMPs reduce EC proliferation and new vessel formation (22). However, Taraboletti and coworkers demonstrated that EMPs isolated from human umbilical vein ECs at low levels promoted formation of capillary-like structures by ECs, whereas high levels abolished angiogenesis (18). Aharon et al. observed that microvesicles which bore MMP played a key role in angiogenesis and were involved in EC invasion and capillary formation (11). It is suggested that EMPs might be indicators of prognosis in patients with cancer $(12,23)$. Consequently, the exact role of EMPs in cancer, and particularly in angiogenesis, needs to be further investigated. There is an increasing number of studies investigating the influence of chemotherapy on EMPs, however, data on the interplay between ionizing radiation and EMPs is very limited (21). An in vitro study by Neuber et al. showed that X-ray doses of 2 and 4 Gy tended to increase the release of CD146positive EMPs from human aortic ECs, however, the finding was not statistically significant (24). In vitro EMP release was also reported in response to irradiation with gamma ray at $2 \mathrm{~Gy}$, but not to protons $(25,26)$. Our study did not show increased EMP numbers after RT/RCT compared to pretreatment levels. This might be attributable to the small study group or EMP numbers being significantly higher primarily in irradiated tissue and not in peripheral blood, which might explain the dissonance from the outcomes of in vitro studies.

VEGF is a signal protein produced by cells that stimulates vasculogenesis and angiogenesis. Cancer cells can express VEGF, which contributes to more effective tumor growth and metastasis by the formation of new vessels and by providing adequate blood supply (27). Many studies demonstrated that expression of VEGF is correlated with poor prognosis, which suggests that the plasma level of VEGF might be a prognostic marker of cancer (14).

EMPs have been reported to have a high angiogenic activity in vitro and in vivo (in a mouse model) through stimulation of microvascular ECs and pericytes to form new vessels (28). This could be of a great significance for cancer growth and metastasis. In this study, we investigated the contribution of EMPs to blood vessel formation after RT as a stimulus by measuring the correlation between EMPs and VEGF levels. Our findings do not support the assumption that EMPs participate in the process of neovascularization since no correlation between EMPs and VEGF levels was identified. However, this study did find that the VEGF level increased after RT and decreased 3 months after treatment, which suggests that RT affects the VEGF concentration in plasma of patients with HNC. The overexpression of VEGF induced by RT was reported e.g. in hepatocellular carcinoma (15) and glioblastoma multiforme (29). Although crucial for healthy tissue repair post-RT, VEGF secretion can also contribute to radioresistance, which provides a rationale for the combination of RT and anti-VEGF agents in patients with HNC (30). 
Summarizing, EMPs are considered complex structures which express a large variety of endothelial molecules. In the literature, the occurrence of EMPs in disease has been associated with endothelial impairment (31). Many researchers have studied various types of cell-derived vesicles, but EMPs have gained special clinical attention. The view of EMPs has changed over the years from mere artifacts called "dust" to active particles in blood coagulation, inflammation and angiogenesis. In conclusion, the present data do not support our hypothesis that RT/RCT influences rapid EMP release, thereby contributing directly to blood vessel formation in patients with HNC. Further research on EMPs should focus on gathering a larger study group to define whether plasma numbers of EMPs in patients with $\mathrm{HNC}$ are sufficient to stimulate angiogenesis.

\section{Conflicts of Interest}

The Authors declare no conflicts of interest in regard to this study.

\section{References}

1 Sierko E, Sokół $M$ and Wojtukiewicz MZ: Endothelial microparticles (EMPs) in physiology and pathology. Postepy Hig Med Dosw 69: 925-932, 2015.

2 Herring JM, McMichael MA and Smith SA: Microparticles in health and disease. J Vet Intern Med 27: 1020-1033, 2013.

3 Leroyer AS, Anfosso F, Lacroix R, Sabatier F, Simoncini S, Njock SM, Jourde N, Brunet P, Camoin-Jau L, Sampol J and Dignat-George F: Endothelial-derived microparticles. Biological conveyors at the cross road of inflammation, thrombosis and angiogenesis. Thromb Haemost 104: 456-463, 2010.

4 Jimenez JJ, Jy W, Mauro LM, Soderland C, Horstman LL and Ahn YS: Endothelial cells release phenotypically and quantitatively distinct microparticles in activation and apoptosis. Thromb Res 109: 175-180, 2003.

5 Amabile N, Guerin AP, Leroyer A, Mallat Z, Nguyen C, Boddaert J, London GM, Tedgui A and Boulanger CM: Circulating endothelial microparticles are associated with vascular dysfunction in patients with end-stage renal failure. $\mathrm{J}$ Am Soc Nephrol 16: 3381-3388, 2005.

6 Piccin A: Endothelial microparticles and endothelial damage: 'The Tip and the iceberg'. Acta Haematol 132:199-204, 2014.

7 Jimenez J, Jy W, Mauro LM, Horstman LL, Ahn ER, Ahn YS and Minagar A: Elevated endothelial microparticle-monocyte complexes induced by multiple sclerosis plasma and the inhibitory effects of interferon-beta $1 \mathrm{~b}$ on release of endothelial microparticles, formation and transendothelial migration of monocyte-endothelial microparticle complexes. Mult Scler 11: 310-315, 2005.

8 Chirinos JA, Heresi GA, Velasquez H, Jy W, Jimenez JJ, Ahn E, Horstman LL, Soriano AO, Zambrano JP and Ahn YS: Elevation of endothelial microparticles, platelets, and leukocyte activation in patients with venous thromboembolism. J Am Coll Cardiol 45: 1467-1471, 2005.

9 Markiewicz M, Richard E, Marks N and Ludwicka-Bradley A: Impact of endothelial microparticles on coagulation, inflammation, and angiogenesis in age-related vascular diseases. J Aging Res 20: 734509, 2013.
10 Combes V, Simon AC, Grau GE, Arnoux D, Camoin L, Sabatier F, Mutin M, Sanmarco M, Sampol J and Dignat-George F: In vitro generation of endothelial microparticles and possible prothrombotic activity in patients with lupus anticoagulant J Clin Invest 104: 93-102, 1999.

11 Aharon A and Brenner B: Microparticles, thrombosis and cancer. Best Pract \& Res Clin Hematol 22: 61-69, 2009.

12 Fleitas T, Martínez-Sales V, Vila V, Reganon E, Mesado D, Martín M, Gómez-Codina J, Montalar J and Reynés G: Circulating ECs and microparticles as prognostic markers in advanced non-small cell lung cancer. PLoS One 10: e47365, 2012.

13 Bruyndonckx L, Hoymans VY, Frederix G, De Guchtenaere A, Franckx H, Vissers DK, Vrints CJ, Ramet J and Conraads VM: Endothelial progenitor cells and endothelial microparticles are independent predictors of endothelial function. J Pediatr 165: 300-305, 2014.

14 Poon RT, Fan ST and Wong J: Clinical implications of circulating angiogenic factors in cancer patients. J Clin Oncol 19: 1207-1225, 2001.

15 Chung YL, Jian JJ, Cheng SH, Tsai SY, Chuang VP, Soong T, Lin YM and Horng CF: Sublethal irradiation induces vascular endothelial growth factor and promotes growth of hepatoma cells: Implications for radiotherapy of hepatocellular carcinoma. Clin Cancer Res 12: 2706-2715, 2006.

16 Lechner D and Weltermann A: Chemiotherapy-induced thrombosis: A role for microparticles and tissue factors? Semin Thromb Haemost 34: 199-203, 2008.

17 Lechner D, Kollars M, Gleiss A, Kyrle A and Weltermann A: Chemotherapy-induced thrombin generation via procoagulant endothelial microparticles is independent of tissue factor activity. J Thromb Haemost 5: 2445-2452, 2007.

18 Taraboletti G, D'Ascenzo S, Borsotti P, Giavazzi R, Pavan A and Dolo V: Shedding of the matrix metalloproteinasese MMP-2, MMP-9, and MT1-MMP as membrane vesicles-associated components by ECs. Am J Pathol 160: 673-680, 2002.

19 Amabile N, Heiss C, Real WM, Minasi P, McGlothlin D, Rame EJ, Grossman W, De Marco T and Yeghiazarians Y: Circulating endothelial microparticle levels predict hemodynamic severity of pulmonary hypertension. Am J Respir Crit Care Med 177: 1268-1275, 2008.

20 Edge SB, Byrd DR, Compton CC, Fritz AG, Greene FL and Trotti A (editors): AJCC Cancer Staging Manual, Seventh edition. France: Springer; 2010. Available from: https://cancerstaging.org/ references-tools/deskreferences/Documents/AJCC $\% 207$ th\% 20Ed\%20Cancer\%20Staging\%20Manual.pdf

21 Yoshimura M, Itasaka S, Harada H and Hiraoka M: Microenvironment and radiation therapy. Biomed Res Int 2013: 685308, 2013.

22 Mezentsev A, Merks RMH, O'Riordan E, Chen J, Mendelev N, Goligorsky MS and Brodsky SV: Endothelial microparticles affect angiogenesis in vitro: Role of oxidative stress. Am J Physiol Heart Circ Physiol 289: H1106-H1114, 2005.

23 García Garre E, Luengo Gil G, Montoro García S, Gonzalez Billalabeitia E, Zafra Poves M, García Martinez E, Roldán Schilling V, Navarro Manzano E, Ivars Rubio A, Lip GYH and Ayala de la Peña F: Circulating small-sized endothelial microparticles as predictors of clinical outcome after chemotherapy for breast cancer: An exploratory analysis. Breast Cancer Res Treat 169: 83-92, 2018. 
24 Neuber C, Pufe J and Pietzsch J: Influence of irradiation on release of endothelial microparticles (EMPs) in vitro. Clin Hemorheol Microcirc 61: 291-299, 2015.

25 Sharma P, Templin T and Grabham P: Short term effects of gamma radiation on endothelial barrier function: Uncoupling of PECAM-1. Microvasc Res 86: 11-20, 2013.

26 Templin T, Sharma P, Guida P and Grabham P: Short-term effects of low-LET radiation on the endothelial barrier: Uncoupling of PECAM-1 and the production of endothelial microparticles. Radiat Res 186: 602-613, 2016.

27 Bergers $G$ and Benjamin LE: Tumorigenesis and the angiogenic switch. Nat Rev Cancer 3: 401-410, 2003.

28 Arderiu G, Peña E and Badimon L: Angiogenic microvascular ECs release microparticles rich in tissue factor that promotes postischemic collateral vessel formation. Arterioscler Thromb Vasc Biol 35: 348-357, 2015.

29 Hovinga KE, Stalpers LJ, van Bree C, Donker M, Verhoeff JJ, Rodermond HM, Bosch DA and van Furth WR: Radiationenhanced vascular endothelial growth factor (VEGF) secretion in glioblastoma multiforme cell lines - a clue to radioresistance? J Neurooncol 74: 99-103, 2005.
30 Lee NY, Zhang Q, Pfister DG, Kim J, Garden AS, Mechalakos J, Hu K, Le QT, Colevas AD, Glisson BS, Chan AT and Ang KK: Phase II study of the addition of bevacizumab to standard chemoradiation for loco-regionally advanced nasopharyngeal carcinoma: Radiation Therapy Oncology Group (RTOG) Trial 0615. Lancet Oncol 13: 172-180, 2012.

31 Lovren $\mathrm{F}$ and Verma S: Evolving role of microparticles in the pathophysiology of endothelial dysfunction. Clin Chem 59: 1166-1174, 2013.

Received December 9, 2018

Revised December 29, 2018

Accepted January 10, 2019 